\title{
The Incidence and Outcomes of Healthcare- associated Respiratory Tract Infections in Non- ventilated Neurocritical Care Patients: Results of a 10-year Prospective Cohort Study
}

Ksenia Ershova ( $\sim$ kseniae@uw.edu )

Skolkovo Institute of Science and Technology https://orcid.org/0000-0002-9877-4471

Ivan Savin

Burdenko National Medical Research Center of Neurosurgery

Oleg Khomenko

Skolkovo Institute of Science and Technology: Skolkovskij institut nauki i tehnologij

Darren Wong

University of Southern California Keck School of Medicine

Gleb Danilov

Burdenko National Medical Research Center of Neurosurgery

Michael Shifrin

Burdenko National Medical Research Center of Neurosurgery

Ekaterina Sokolova

Burdenko National Medical Research Center of Neurosurgery

Vikas N O'Reilly-Shah

University of Washington

Abhijit V. Lele

University of Washington

Olga Ershova

Burdenko National Medical Research Center of Neurosurgery

\section{Research}

Keywords: Cross Infection, Pneumonia, Ventilator-Associated, Respiratory Tract Infections, Critical Care Outcomes, Observational Study

Posted Date: July 26th, 2021

DOl: https://doi.org/10.21203/rs.3.rs-719352/v1 
License: (c) (i) This work is licensed under a Creative Commons Attribution 4.0 International License. Read Full License

Version of Record: A version of this preprint was published at Journal of Clinical Neuroscience on March 1st, 2022. See the published version at https://doi.org/10.1016/j.jocn.2021.12.035. 


\section{Abstract}

Background. The incidence of healthcare-associated respiratory tract infections in non-ventilated patients (NVA-HARTI) in neurosurgical intensive care units (ICU) is unknown. The impact of NVA-HARTI on patient outcomes and differences between NVA-HARTI and ventilator-associated healthcare-associated respiratory tract infections (VA-HARTI) are poorly understood. Our objectives were to report the incidence, hospital length of stay (LOS), ICU LOS, and mortality in neurosurgical ICU and compare these characteristics between NVA- and VA-HARTI.

Methods. This prospective cohort study was conducted in a neurosurgical ICU in Moscow from 2011 to 2020. All patients with ICU LOS $>48 \mathrm{~h}$ were included. Time trends were analyzed for all outcomes. A competing risk model was used for survival and risk analysis.

Results. A total of 3,937 ICU admissions were analyzed. NVA-HARTI vs VA-HARTI results were: cumulative incidence 7.2 (6.4-8.0) vs 15.4 (14.2-16.5) per 100 ICU admissions, incidence rate $4.2 \pm 2.0$ vs $9.5 \pm 3.0$ per 1000 patient-days in the ICU, median LOS 32 [21; 48.5] vs 46 [28; 76.5] days, median ICU LOS 15 [10; $28.75]$ vs 26 [17; 43] days, and mortality rates $12.3 \%$ (7.9-16.8) vs $16.7 \%$ (13.6-19.7). The incidence of VAHARTI decreased in ten years while NVA-HARTI incidence did not change. VA-HARTI was found to be an independent risk factor of death, odds ratio 1.54 (1.11-2.14), p-value=0.009 while NVA-HARTI was not.

Conclusion. Our findings suggest that NVA-HARTI in neurosurgical ICU patients represents a significant healthcare burden with relatively high incidence and associated poor outcomes. NVA-HARTI appeared to be different from VA-HARTI and persisted despite preventive measures; therefore, extrapolating VA-HARTI research findings to NVA-HARTI should be avoided.

\section{Background}

Healthcare-associated respiratory tract infections (HARTI) in intensive care units (ICU) are typically associated with mechanical ventilation comprising a clinical entity of ventilator-associated HARTI (VAHARTI). However, non-ventilated patients can also develop HARTI, non-ventilator associated HARTI (NVAHARTI). While ventilator-associated infections, especially ventilator-associated pneumonia (VAP), have been extensively studied and their incidence, risk factors, and outcomes widely reported $(1,2)$, very little in this regard is known about NVA-HARTI in ICU patients $(3,4)$, and some scientists call it a 'neglected disease' (5). Simultaneously, according to the most recent USA point prevalence survey, pneumonia in non-ventilated patients constitutes the majority of healthcare-associated pneumonia cases in U.S. hospitals (6). Additionally, the association has been shown between NVA-HARTI and increased risk of 30day ICU mortality (7). Given potentially poor outcomes and high prevalence, the medical community called for more research in the area of NVA-HARTI (8).

To our knowledge, the incidence of NVA-HARTI remains largely unknown for neurosurgical ICU patients. However, these patients are at higher risk of HARTI given high prevalence of factors predisposing them to respiratory tract colonization, such as altered consciousness and insufficient cough. Additionally, it is 
unclear whether VA- and NVA-HARTI are similar or different clinical categories (8) and whether it is warranted to extrapolate results from VAP research to NVA-HARTI. Therefore, our primary objective was to evaluate the incidence, prevalence, risks, and outcomes of NVA-HARTI in neurosurgical ICU patients. Our secondary objective was to compare these characteristics between NVA- and VA-HARTI within the same patient population and timeframe.

\section{Methods}

\section{Study Design and Settings}

This prospective cohort study was conducted at the national referral neurosurgical hospital, Burdenko National Medical Research Center of Neurosurgery (NSI) in Moscow, from 2011 to 2020. The hospital has an annual patient flow of approximately 9000 patients, with $98 \%$ undergoing neurosurgical procedures. The ICU at NSI is combined with the post-operative care unit and has a total of 38 beds. On average, 4000 patients are admitted every year to the ICU, $10-13 \%$ stay for $>48$ hours.

\section{Patients and HARTI Definitions}

Patients of all ages admitted to the ICU between January 1, 2011, and December 31, 2020, with a length of ICU stay (ICU LOS) greater than 48 hours were included in the study, excluding those with HARTI present on admission (POA) and tested positive for COVID-19 at any point during their hospital stay. HARTI POA was identified based on the 2019 CDC/NHSN definition (9).

The 2008 CDC/NHSN definition (10) was used in our study to define healthcare-associated infections (HAI); HARTI was defined as a subset of HAl that included all respiratory tract infections: upper respiratory tract infections (pharyngitis, laryngitis, epiglottitis), sinusitis, pneumonia, and lower respiratory tract infections other than pneumonia (bronchitis, tracheobronchitis, tracheitis, other infections of the lower respiratory tract). VA-HARTI was defined per the $2008 \mathrm{CDC}$ /NHSN guidelines (10). The presence of HARTI was assessed daily; standard resolution criteria were used to define resolved cases. For more on definitions, see Supplementary Methods.

\section{Data Collection and Processing}

Data was collected as a part of the ICU infection surveillance protocol and stored in the electronic medical record. ICU nursing staff performed surveillance and reported to the infection control team. Study team members (GD, ES, OE) verified data and performed data entry daily. For each patient, we collected 47 characteristics daily, and eight at the end of the hospital stay. Characteristics included demographics, hospital course and outcomes (Table S1).

The data from patients with ICU LOS exceeding 365 days was censored at ICU day 365. In case of readmission, if it took place within 24 hours from the discharge, it was considered a continuation of the previous ICU admission. If after 24 hours, then this constituted a new ICU admission.

\section{Outcome Measures}


The outcomes of the study included daily prevalence, cumulative incidence, incidence rates, time-risk assessment, and patient outcomes (hospital LOS, ICU LOS, and crude in-hospital mortality). The daily prevalence of HARTI was calculated by dividing the number of patients with active HARTI symptoms in the morning of each day by the total number of patients in the ICU who were enrolled in the study on the same morning. The daily prevalence was reported as a percentage. The cumulative incidence was reported as the number of HARTI cases per $100 \mathrm{ICU}$ admissions. Incidence rates were defined as the number of HARTI cases per 1000 patient-days in the ICU. For VA-HARTI, the incidence rate was also adjusted to days on mechanical ventilation and reported as the number of VA-HARTI cases per 1000 ventilator-days. The results of the time-risk assessment were reported as a cumulative probability and a daily risk (instantaneous hazard).

The hospital LOS and ICU LOS were reported in days. The crude in-hospital mortality was defined as death from any cause occurring during the hospital stay. The hospital LOS and mortality were attributed to a particular group of patients based on a group hierarchy: 'Dual HARTI' > 'VA-HARTI' > 'NVA-HARTI' > 'Other HAl' > 'No HAl'. A patient was excluded from other groups where he/she might be counted based on his/her other ICU admissions.

\section{Statistical analysis}

The database was extracted and de-identified. The percentage of missing values was calculated for each variable; missing values were treated using manual fill, forward-fill or backward-fill where applicable (Table S2). Data cleaning did not include any data transformation or outlier removal. According to the first objective of the study, we calculated the mean incidence, prevalence, risks, and outcomes of NVA-HARTI and their dynamics over the study period. Binary measurements were reported as a number of events with percentage and a $95 \%$ confidence interval $(\mathrm{Cl})$ for binomial distribution. Continuous and ordinal measurements were reported as a median with first and third quartiles $(\mathrm{Q} 1 ; \mathrm{Q} 3)$ or as a mean with standard deviation. The linear regression and Augmented Dickey-Fuller test (ADF) test were used to evaluate time trends. Monthly, quarterly, or yearly data was used depending on the model. For each variable, the model was checked for relevance based on the distribution of residuals and QQ plot. In the $A D F$ test for stationarity, the variable's dynamic was considered non-stationary if mean, standard deviation, or frequency changed over time; non-stationarity was assumed as a null hypothesis. To extract a trend from prevalence data, we used the additive decomposition method defined as a centered moving average of the data within the nearest $n$ periods, assuming that the seasonal component is constant from year to year.

Included ICU admissions were divided in five groups depending on the presence and type of infection: VAHARTI, NVA-HARTI, Other HAI, No HAI, and the group of patients who had both types of HARTI during a single ICU admission ('Dual HARTI') (Fig. 1, groups shown in blue). We excluded the Dual HARTI group from outcome analysis as an outlier with an insufficient sample size. The null hypothesis of the second objective of the study was set as a lack of difference between VA- and NVA-HARTI groups for all outcomes. The Kruskal-Wallis test was used to compare continuous variables among three or more groups with post-hoc pairwise comparison by Conover test. To compare the distribution of categorical 
variables among two or more groups, we used a chi-square test with a post-hoc pairwise chi-square test when needed. P-values were adjusted for multiple comparisons by the Bonferroni-Holm method if $>5$ hypotheses were tested.

To compare mortality between groups, we used a competing risk model of a sub-distribution function (11) implemented in (12) for survival analysis; a discharge from the hospital was considered a competing event. For additional considerations regarding the methodology of survival analysis, see Supplementary

\section{Methods.}

The immortal-time bias was present; we utilized two approaches to account for it. First, we used the Cox time varying proportional hazard model to account for time-independent variables (age, sex, Charlson Comorbidity Index and admitting diagnosis). To account for all confounding variables (time-dependent and time-independent), we used logistic regression. Both regressions were applied separately for VA- and NVA-HARTI data. The procedure for the logistic regression was as follows. First, the univariate analysis was carried out comparing all factors between patients with and without an infection. Factors with a pvalue $<0.05$ after adjustment for multiple comparisons were entered into the first logistic regression. Variables that demonstrated $p$-value $>0.05$ (were not independent predictors of infection) and a binary infection variable were used in the second logistic regression to evaluate whether they can independently predict mortality. Model performance was assessed in five-fold cross-validation with a receiving-operating curve, an area under the curve (ROC-AUC) score used as a performance metric.

The HARTI time-risk assessment was performed using a competing risk model; discharge from the hospital and death were considered competing events. Kernel-smoothed instantaneous (daily) hazard of infection was obtained using a Nelson-Aalen estimator.

The significance level was set at 0.05 . Statistical analysis was performed in Python 3.7 using StatsModels (13) and Scipy (14) libraries.

\section{Results}

\section{Study population}

A total of 3,842 unique patients accounting for 4,258 ICU admissions were admitted for greater than 48 hours; 310 ICU admissions were excluded due to HARTI present on admission, and 11 ICU admissions were excluded due to positive COVID-19 test. The final data sample of 3,937 ICU admissions was analyzed (Fig. 1).

\section{Characteristics of NVA and VA-HARTI}

The univariate analysis showed that NVA- and VA-HARTI patients were similar in terms of patients' demographics, admitting diagnosis, surgeries, and complications, although their ICU courses and severity were different (Table 1). When comparing five groups, there were significant differences among them, most notably in their ICU course and complications (Table S3). We also found that patient characteristics 
remained mostly stable during ten years while multiple aspects of medical and surgical practice have changed. Most notably, the use of mechanical ventilation, benzodiazepines, urinary catheters, and antibiotics decreased significantly (Table S4). 
Table 1

Baseline clinical characteristics of NVA-HARTI and VA-HARTI patients; for baseline characteristics of other patient groups refer to Table S3. The p-values are obtained from t-test test for numerical variables and Chi-square test for categorical variables. Bonferroni-Holm correction for multiple comparisons was used to adjust $p$-values. Yellow highlighting indicates characteristics with $p$-value below the level of significance. Abbreviations: $\mathrm{Cl}$, confidence interval; CNS, central nervous system; CSFL-NE, CSF leakage from nose and/or ears; CSFL-SS, CSF leakage from surgical site; EVD, external ventricular drain; EETS, Endoscopic Endonasal Trans-sphenoidal Surgery; HAl, healthcare-associated infections; ICU, intensive care unit; ICP, intracranial pressure; INSD, Implantation or Revision of a Neurosurgical Device; NVA-HARTI, non-ventilator-associated healthcare-associated respiratory tract infections; PBSS, Pittsburgh Brain Stem

Score; RASS, Richmond Agitation-Sedation Scale, SSSI, superficial surgical site infections; VA-HARTI, ventilator-associated healthcare-associated respiratory tract infections.
NVA-HARTI $(n=$
VA-HARTI $(n=577)$
p-value
Adjusted p- 254)

$\begin{array}{llll}\begin{array}{l}\text { No of } \\ \text { patients }\end{array} & \begin{array}{l}95 \% \mathrm{Cl} \\ \text { or } 1 \mathrm{st}\end{array} & \begin{array}{l}\text { No of } \\ \text { patients }\end{array} & \begin{array}{l}95 \% \mathrm{Cl} \text { or } 1 \mathrm{st} \\ \text { and 3rd }\end{array} \\ \begin{array}{l}\text { (\%) or } \\ \text { median }\end{array} & \begin{array}{l}\text { and } 3 \mathrm{rd} \\ \text { quartiles }\end{array} & \begin{array}{l}\text { median } \\ \text { quartiles }\end{array} & \end{array}$

\section{Demographics}

$\begin{array}{lllllll}\text { Age, years } & 52.0 & \begin{array}{l}{[31.25 ;} \\ 62.0]\end{array} & 47.0 & {[31.0 ; 60.0]} & 0.06101 & 0.88955 \\ \text { Male patients } & \begin{array}{l}153 \\ (60.2 \%)\end{array} & \begin{array}{l}{[54.2 ;} \\ 66.3]\end{array} & \begin{array}{l}310 \\ (53.7 \%)\end{array} & {[49.7 ; 57.8]} & 0.09597 & 0.96763 \\ & 3.0 & {[2.0 ; 5.0]} & 3.0 & {[2.0 ; 5.0]} & 0.10293 & 0.96763 \\ \begin{array}{l}\text { Charlson } \\ \begin{array}{l}\text { Comorbidity } \\ \text { Index }\end{array}\end{array} & & & & & \end{array}$

Admitting diagnosis

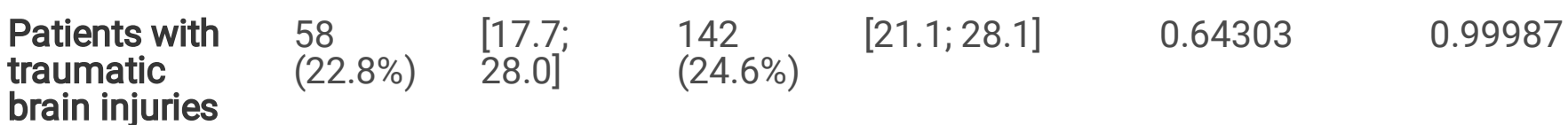

\begin{tabular}{|c|c|c|c|c|c|c|}
\hline $\begin{array}{l}\text { Patients with } \\
\text { brain tumors }\end{array}$ & $\begin{array}{l}127 \\
(50.0 \%)\end{array}$ & $\begin{array}{l}{[43.9 ;} \\
56.1]\end{array}$ & $\begin{array}{l}278 \\
(48.2 \%)\end{array}$ & {$[44.1 ; 52.3]$} & 0.68315 & 0.99987 \\
\hline $\begin{array}{l}\text { Patients with } \\
\text { neurovascular } \\
\text { disorders }\end{array}$ & $\begin{array}{l}59 \\
(23.2 \%)\end{array}$ & $\begin{array}{l}{[18.0 ;} \\
28.4]\end{array}$ & $\begin{array}{l}142 \\
(24.6 \%)\end{array}$ & {$[21.1 ; 28.1]$} & 0.73342 & 0.99987 \\
\hline $\begin{array}{l}\text { Patients with } \\
\text { other } \\
\text { neurosurgical } \\
\text { disorders }\end{array}$ & $\begin{array}{l}10 \\
(3.9 \%)\end{array}$ & {$[1.5 ; 6.3]$} & $\begin{array}{l}15 \\
(2.6 \%)\end{array}$ & {$[1.3 ; 3.9]$} & 0.41261 & 0.99975 \\
\hline
\end{tabular}

Surgeries

$\begin{array}{lllllll}\begin{array}{l}\text { Patients } \\ \text { undergoing }\end{array} & 80 & {[25.8 ;} & 253 & {[39.8 ; 47.9]} & 0.00107 & 0.04925 \\ \text { INSD } & 37.5 \%) & (43.8 \%) & & & \end{array}$




\begin{tabular}{|c|c|c|c|c|c|c|}
\hline & \multicolumn{2}{|c|}{$\begin{array}{l}\text { NVA-HARTI }(n= \\
254)\end{array}$} & \multicolumn{2}{|c|}{ VA-HARTI $(n=577)$} & \multirow[t]{2}{*}{ p-value } & \multirow[t]{2}{*}{$\begin{array}{l}\text { Adjusted p- } \\
\text { value }\end{array}$} \\
\hline & $\begin{array}{l}\text { No of } \\
\text { patients } \\
(\%) \text { or } \\
\text { median }\end{array}$ & $\begin{array}{l}95 \% \mathrm{Cl} \\
\text { or } 1 \mathrm{st} \\
\text { and } 3 \mathrm{rd} \\
\text { quartiles }\end{array}$ & $\begin{array}{l}\text { No of } \\
\text { patients } \\
\text { (\%) or } \\
\text { median }\end{array}$ & $\begin{array}{l}95 \% \mathrm{Cl} \text { or } 1 \mathrm{st} \\
\text { and } 3 \text { rd } \\
\text { quartiles }\end{array}$ & & \\
\hline $\begin{array}{l}\text { Patients } \\
\text { undergoing } \\
\text { non-cranial } \\
\text { surgeries }\end{array}$ & $\begin{array}{l}119 \\
(46.9 \%)\end{array}$ & $\begin{array}{l}{[40.7} \\
53.0]\end{array}$ & $\begin{array}{l}341 \\
(59.1 \%)\end{array}$ & {$[55.1 ; 63.1]$} & 0.00139 & 0.06076 \\
\hline $\begin{array}{l}\text { Patients } \\
\text { undergoing } \\
\text { craniotomy }\end{array}$ & $\begin{array}{l}172 \\
(67.7 \%)\end{array}$ & $\begin{array}{l}\text { [62.0; } \\
73.5]\end{array}$ & $\begin{array}{l}402 \\
(69.7 \%)\end{array}$ & {$[65.9 ; 73.4]$} & 0.6312 & 0.99987 \\
\hline $\begin{array}{l}\text { Patients } \\
\text { undergoing } \\
\text { endovascular } \\
\text { neurosurgical } \\
\text { procedures }\end{array}$ & $\begin{array}{l}26 \\
(10.2 \%)\end{array}$ & $\begin{array}{l}{[6.5 ;} \\
14.0]\end{array}$ & $\begin{array}{l}70 \\
(12.1 \%)\end{array}$ & {$[9.5 ; 14.8]$} & 0.50304 & 0.99975 \\
\hline $\begin{array}{l}\text { Patients } \\
\text { undergoing } \\
\text { EETS }\end{array}$ & $\begin{array}{l}9 \\
(3.5 \%)\end{array}$ & {$[1.3 ; 5.8]$} & $\begin{array}{l}24 \\
(4.2 \%)\end{array}$ & {$[2.5 ; 5.8]$} & 0.82104 & 0.99987 \\
\hline $\begin{array}{l}\text { Patients } \\
\text { undergoing } \\
\text { spinal surgery }\end{array}$ & $\begin{array}{l}5 \\
(2.0 \%)\end{array}$ & {$[0.3 ; 3.7]$} & $\begin{array}{l}17 \\
(2.9 \%)\end{array}$ & {$[1.6 ; 4.3]$} & 0.56576 & 0.99976 \\
\hline $\begin{array}{l}\text { The total } \\
\text { number of all } \\
\text { surgeries } \\
\text { during one } \\
\text { ICU } \\
\text { admission }\end{array}$ & 2.0 & {$[1.0 ; 3.0]$} & 2.0 & {$[1.0 ; 3.0]$} & $<0.0001$ & 0.00173 \\
\hline $\begin{array}{l}\text { The total } \\
\text { duration of all } \\
\text { craniotomies } \\
\text { performed } \\
\text { during one } \\
\text { admission, } \\
\text { minutes }\end{array}$ & 300.0 & $\begin{array}{l}{[240.0 ;} \\
370.0]\end{array}$ & 320.0 & {$[240.0 ; 440.0]$} & 0.0982 & 0.96763 \\
\hline $\begin{array}{l}\text { The total } \\
\text { duration of all } \\
\text { INSDs } \\
\text { performed } \\
\text { during one } \\
\text { admission, } \\
\text { minutes }\end{array}$ & 160.0 & $\begin{array}{l}{[90.0 ;} \\
200.0]\end{array}$ & 150.0 & {$[90.0 ; 247.5]$} & 0.77106 & 0.99987 \\
\hline
\end{tabular}




\begin{tabular}{|c|c|c|c|c|c|c|}
\hline & \multicolumn{2}{|c|}{$\begin{array}{l}\text { NVA-HARTI }(n= \\
254)\end{array}$} & \multicolumn{2}{|c|}{ VA-HARTI $(n=577)$} & \multirow[t]{2}{*}{ p-value } & \multirow[t]{2}{*}{$\begin{array}{l}\text { Adjusted p- } \\
\text { value }\end{array}$} \\
\hline & $\begin{array}{l}\text { No of } \\
\text { patients } \\
(\%) \text { or } \\
\text { median }\end{array}$ & $\begin{array}{l}95 \% \mathrm{Cl} \\
\text { or } 1 \mathrm{st} \\
\text { and } 3 \mathrm{rd} \\
\text { quartiles }\end{array}$ & $\begin{array}{l}\text { No of } \\
\text { patients } \\
(\%) \text { or } \\
\text { median }\end{array}$ & $\begin{array}{l}95 \% \mathrm{Cl} \text { or } 1 \mathrm{st} \\
\text { and } 3 \text { rd } \\
\text { quartiles }\end{array}$ & & \\
\hline $\begin{array}{l}\text { The total } \\
\text { duration of all } \\
\text { EETSs } \\
\text { performed } \\
\text { during one } \\
\text { admission, } \\
\text { minutes }\end{array}$ & 270.0 & $\begin{array}{l}{[215.0 ;} \\
341.25]\end{array}$ & 230.0 & {$[115.0 ; 315.0]$} & 0.2878 & 0.99943 \\
\hline $\begin{array}{l}\text { The total } \\
\text { duration of all } \\
\text { endovascular } \\
\text { neurosurgical } \\
\text { procedures } \\
\text { performed } \\
\text { during one } \\
\text { admission, } \\
\text { minutes }\end{array}$ & 135.0 & $\begin{array}{l}{[90.0 ;} \\
240.0]\end{array}$ & 140.0 & {$[90.0 ; 260.0]$} & 0.92609 & 0.99987 \\
\hline $\begin{array}{l}\text { The total } \\
\text { duration of all } \\
\text { non-cranial } \\
\text { surgeries } \\
\text { performed } \\
\text { during one } \\
\text { admission, } \\
\text { minutes }\end{array}$ & 192.5 & $\begin{array}{l}{[101.25} \\
267.5]\end{array}$ & 187.5 & {$[150.0 ; 278.75]$} & 0.43105 & 0.99975 \\
\hline $\begin{array}{l}\text { The total } \\
\text { duration of all } \\
\text { spinal } \\
\text { surgeries } \\
\text { performed } \\
\text { during one } \\
\text { admission, } \\
\text { minutes }\end{array}$ & 345.0 & $\begin{array}{l}{[307.5 ;} \\
390.0]\end{array}$ & 240.0 & {$[215.0 ; 392.5]$} & 0.35991 & 0.99975 \\
\hline $\begin{array}{l}\text { The total } \\
\text { duration of all } \\
\text { surgeries } \\
\text { during one } \\
\text { ICU } \\
\text { admission }\end{array}$ & 318.5 & $\begin{array}{l}{[226.0 ;} \\
435.5]\end{array}$ & 346.0 & {$[224.0 ; 497.0]$} & 0.11787 & 0.97678 \\
\hline \multicolumn{7}{|c|}{ Severity of patient condition during ICU stay } \\
\hline $\begin{array}{l}\text { Glasgo Coma } \\
\text { Scale }\end{array}$ & 10.0 & $\begin{array}{l}{[7.0 ;} \\
12.0]\end{array}$ & 7.0 & {$[7.0 ; 10.0]$} & $<0.0001$ & $<0.0001$ \\
\hline RASS & -2.0 & $\begin{array}{l}{[-2.0 ;} \\
1.0]\end{array}$ & -2.0 & {$[-2.0 ; 1.0]$} & 0.00226 & 0.09459 \\
\hline
\end{tabular}




\begin{tabular}{|c|c|c|c|c|c|c|}
\hline & \multicolumn{2}{|c|}{$\begin{array}{l}\text { NVA-HARTI }(n= \\
254)\end{array}$} & \multicolumn{2}{|c|}{ VA-HARTI $(n=577)$} & \multirow[t]{2}{*}{ p-value } & \multirow[t]{2}{*}{$\begin{array}{l}\text { Adjusted p- } \\
\text { value }\end{array}$} \\
\hline & $\begin{array}{l}\text { No of } \\
\text { patients } \\
(\%) \text { or } \\
\text { median }\end{array}$ & $\begin{array}{l}95 \% \mathrm{Cl} \\
\text { or } 1 \mathrm{st} \\
\text { and } 3 \mathrm{rd} \\
\text { quartiles }\end{array}$ & $\begin{array}{l}\text { No of } \\
\text { patients } \\
(\%) \text { or } \\
\text { median }\end{array}$ & $\begin{array}{l}95 \% \mathrm{Cl} \text { or } 1 \mathrm{st} \\
\text { and 3rd } \\
\text { quartiles }\end{array}$ & & \\
\hline PBSS & 11.0 & $\begin{array}{l}{[10.0 ;} \\
11.0]\end{array}$ & 11.0 & {$[10.0 ; 11.0]$} & $<0.0001$ & $<0.0001$ \\
\hline \multicolumn{7}{|l|}{ ICU course } \\
\hline $\begin{array}{l}\text { Days with } \\
\text { antibiotics }\end{array}$ & 14.0 & $\begin{array}{l}{[8.0 ;} \\
27.0]\end{array}$ & 24.0 & {$[15.0 ; 42.0]$} & $<0.0001$ & $<0.0001$ \\
\hline $\begin{array}{l}\text { Days with } \\
\text { anxiolytics }\end{array}$ & 0.0 & {$[0.0 ; 2.0]$} & 0.0 & {$[0.0 ; 1.0]$} & 0.33964 & 0.99975 \\
\hline $\begin{array}{l}\text { Days with } \\
\text { arterial line }\end{array}$ & 0.0 & {$[0.0 ; 6.0]$} & 6.0 & {$[0.0 ; 11.0]$} & $<0.0001$ & $<0.0001$ \\
\hline $\begin{array}{l}\text { Days with } \\
\text { central line }\end{array}$ & 11.0 & $\begin{array}{l}\text { [7.0; } \\
21.0]\end{array}$ & 19.0 & {$[13.0 ; 32.0]$} & $<0.0001$ & $<0.0001$ \\
\hline $\begin{array}{l}\text { Days with } \\
\text { EVD }\end{array}$ & 0.0 & {$[0.0 ; 2.0]$} & 0.0 & {$[0.0 ; 7.0]$} & 0.01494 & 0.43557 \\
\hline $\begin{array}{l}\text { Days with } \\
\text { feeding tube }\end{array}$ & 12.0 & $\begin{array}{l}{[7.0 ;} \\
25.5]\end{array}$ & 23.0 & {$[14.0 ; 41.0]$} & $<0.0001$ & $<0.0001$ \\
\hline $\begin{array}{l}\text { Patients with } \\
\text { hypothermia }\end{array}$ & $\begin{array}{l}2 \\
(0.8 \%)\end{array}$ & {$[0.0 ; 1.9]$} & $37(6.4 \%)$ & {$[4.4 ; 8.4]$} & 0.0008 & 0.03751 \\
\hline $\begin{array}{l}\text { Patients with } \\
\text { ICP } \\
\text { monitoring }\end{array}$ & $\begin{array}{l}54 \\
(21.3 \%)\end{array}$ & $\begin{array}{l}{[16.2 ;} \\
26.3]\end{array}$ & $228(39.5 \%)$ & {$[35.5 ; 43.5]$} & $<0.0001$ & $<0.0001$ \\
\hline $\begin{array}{l}\text { Patients with } \\
\text { mechanical } \\
\text { ventilation }\end{array}$ & $\begin{array}{l}229 \\
(90.2 \%)\end{array}$ & $\begin{array}{l}\text { [86.5; } \\
93.8]\end{array}$ & $577(100.0 \%)$ & {$[100.0 ; 100.0]$} & $<0.0001$ & $<0.0001$ \\
\hline $\begin{array}{l}\text { Patients with } \\
\text { mutism }\end{array}$ & $\begin{array}{l}40 \\
(15.7 \%)\end{array}$ & $\begin{array}{l}\text { [11.3; } \\
20.2]\end{array}$ & $144(25.0 \%)$ & {$[21.4 ; 28.5]$} & 0.00431 & 0.16943 \\
\hline $\begin{array}{l}\text { Patients with } \\
\text { persistent } \\
\text { vegetative } \\
\text { state }\end{array}$ & $\begin{array}{l}33 \\
(13.0 \%)\end{array}$ & $\begin{array}{l}{[8.9 ;} \\
17.1]\end{array}$ & 154 (26.7\%) & {$[23.1 ; 30.3]$} & $<0.0001$ & 0.00109 \\
\hline $\begin{array}{l}\text { Patients with } \\
\text { sedation }\end{array}$ & $\begin{array}{l}142 \\
(55.9 \%)\end{array}$ & $\begin{array}{l}{[49.8 ;} \\
62.0]\end{array}$ & 395 (68.5\%) & {$[64.7 ; 72.2]$} & 0.00066 & 0.03162 \\
\hline $\begin{array}{l}\text { Patients with } \\
\text { seizures }\end{array}$ & $\begin{array}{l}22 \\
(8.7 \%)\end{array}$ & $\begin{array}{l}{[5.2 ;} \\
12.1]\end{array}$ & 49 (8.5\%) & {$[6.2 ; 10.8]$} & 0.9567 & 0.99987 \\
\hline
\end{tabular}




\begin{tabular}{|c|c|c|c|c|c|c|}
\hline & \multicolumn{2}{|c|}{$\begin{array}{l}\text { NVA-HARTI }(n= \\
254)\end{array}$} & \multicolumn{2}{|c|}{ VA-HARTI $(n=577)$} & \multirow[t]{2}{*}{ p-value } & \multirow[t]{2}{*}{$\begin{array}{l}\text { Adjusted p- } \\
\text { value }\end{array}$} \\
\hline & $\begin{array}{l}\text { No of } \\
\text { patients } \\
(\%) \text { or } \\
\text { median }\end{array}$ & $\begin{array}{l}95 \% \mathrm{Cl} \\
\text { or } 1 \mathrm{st} \\
\text { and } 3 \mathrm{rd} \\
\text { quartiles }\end{array}$ & $\begin{array}{l}\text { No of } \\
\text { patients } \\
\text { (\%) or } \\
\text { median }\end{array}$ & $\begin{array}{l}95 \% \mathrm{Cl} \text { or } 1 \mathrm{st} \\
\text { and 3rd } \\
\text { quartiles }\end{array}$ & & \\
\hline $\begin{array}{l}\text { Patients with } \\
\text { total } \\
\text { parenteral } \\
\text { feeding }\end{array}$ & $\begin{array}{l}14 \\
(5.5 \%)\end{array}$ & {$[2.7 ; 8.3]$} & $45(7.8 \%)$ & {$[5.6 ; 10.0]$} & 0.30017 & 0.99944 \\
\hline $\begin{array}{l}\text { Patients with } \\
\text { urinary } \\
\text { catheter }\end{array}$ & $\begin{array}{l}238 \\
(93.7 \%)\end{array}$ & $\begin{array}{l}{[90.7 ;} \\
96.7]\end{array}$ & $569(98.6 \%)$ & {$[97.7 ; 99.6]$} & 0.00024 & 0.01225 \\
\hline $\begin{array}{l}\text { Patients with } \\
\text { vasopressors }\end{array}$ & $\begin{array}{l}125 \\
(49.2 \%)\end{array}$ & $\begin{array}{l}{[43.1 ;} \\
55.4]\end{array}$ & 399 (69.2\%) & {$[65.4 ; 72.9]$} & $<0.0001$ & $<0.0001$ \\
\hline \multicolumn{7}{|l|}{ Complications } \\
\hline $\begin{array}{l}\text { Days with } \\
\text { intestinal } \\
\text { dysfunction }\end{array}$ & 0.0 & {$[0.0 ; 4.0]$} & 3.0 & {$[0.0 ; 9.0]$} & $<0.0001$ & $<0.0001$ \\
\hline $\begin{array}{l}\text { Days with } \\
\text { urinary tract } \\
\text { infection }\end{array}$ & 0.0 & {$[0.0 ; 3.0]$} & 2.0 & {$[0.0 ; 14.0]$} & $<0.0001$ & $<0.0001$ \\
\hline $\begin{array}{l}\text { Patients with } \\
\text { bloodstream } \\
\text { infection }\end{array}$ & $\begin{array}{l}24 \\
(9.4 \%)\end{array}$ & $\begin{array}{l}{[5.9 ;} \\
13.0]\end{array}$ & $76(13.2 \%)$ & {$[10.4 ; 15.9]$} & 0.16037 & 0.99108 \\
\hline $\begin{array}{l}\text { Patients with } \\
\text { CNS infection }\end{array}$ & $\begin{array}{l}37 \\
(14.6 \%)\end{array}$ & $\begin{array}{l}{[10.2 ;} \\
18.9]\end{array}$ & $100(17.3 \%)$ & {$[14.2 ; 20.4]$} & 0.37464 & 0.99975 \\
\hline $\begin{array}{l}\text { Patients with } \\
\text { CSFL-NE }\end{array}$ & $\begin{array}{l}42 \\
(16.5 \%)\end{array}$ & $\begin{array}{l}{[12.0 ;} \\
21.1]\end{array}$ & $122(21.1 \%)$ & {$[17.8 ; 24.5]$} & 0.14899 & 0.98908 \\
\hline $\begin{array}{l}\text { Patients with } \\
\text { CSFL-SS }\end{array}$ & $\begin{array}{l}19 \\
(7.5 \%)\end{array}$ & $\begin{array}{l}{[4.2 ;} \\
10.7]\end{array}$ & $62(10.7 \%)$ & {$[8.2 ; 13.3]$} & 0.1819 & 0.99339 \\
\hline $\begin{array}{l}\text { Patients with } \\
\text { intestinal } \\
\text { dysfunction }\end{array}$ & $\begin{array}{l}120 \\
(47.2 \%)\end{array}$ & $\begin{array}{l}{[41.1 ;} \\
53.4]\end{array}$ & $363(62.9 \%)$ & {$[59.0 ; 66.9]$} & $<0.0001$ & 0.00183 \\
\hline $\begin{array}{l}\text { Patients with } \\
\text { other } \\
\text { infections }\end{array}$ & $\begin{array}{l}43 \\
(16.9 \%)\end{array}$ & $\begin{array}{l}\text { [12.3; } \\
\text { 21.5] }\end{array}$ & $144(25.0 \%)$ & {$[21.4 ; 28.5]$} & 0.01379 & 0.41816 \\
\hline $\begin{array}{l}\text { Patients with } \\
\text { SSSI }\end{array}$ & $\begin{array}{l}11 \\
(4.3 \%)\end{array}$ & {$[1.8 ; 6.8]$} & $54(9.4 \%)$ & {$[7.0 ; 11.7]$} & 0.01895 & 0.50729 \\
\hline $\begin{array}{l}\text { Patients with } \\
\text { urinary tract } \\
\text { infection }\end{array}$ & $\begin{array}{l}83 \\
(32.7 \%)\end{array}$ & $\begin{array}{l}{[26.9 ;} \\
38.4]\end{array}$ & $320(55.5 \%)$ & {$[51.4 ; 59.5]$} & $<0.0001$ & $<0.0001$ \\
\hline
\end{tabular}


Of the 3,937 ICU admissions, 859 had at least one episode of HARTI with a total of 955 discrete HARTI cases, group distribution shown at Fig. 1. Recurrent HARTI cases were observed in 79 ICU admissions.

Among patients who had a single HARTI event during their hospital stay, NVA-HARTI occurred on median ICU day 4, with the onset of the half of the cases between ICU days 3 and 7 (Figure S1A). The onset of VAHARTI occurred later, on median ICU day 7 [Q1,Q3: 5, 12] and on median day 6 of mechanical ventilation [Q1,Q3: 4, 9], Figures S1A, S1B. The symptoms of NVA-HARTI were present for a median of 7 days [Q1,Q3: $3,11]$, as compared to 9 days [Q1,Q3: 5, 16] for VA-HARTI (Figure S1C).

\section{Prevalence of HARTI}

The mean daily prevalence of NVA-HARTI was $5.6 \%$ and there was no longitudinal time trend observed, pvalue $=0.85$ (Figure S2A). The mean prevalence of VA-HARTI was $13.7 \%$ and it significantly decreased from 2011 to 2020, p-value $=0.00011$ (Figure S2B). For both infections, the prevalence fluctuated significantly from day to day, and the extracted annual trend had irregular periodic patterns.

\section{Incidence of HARTI}

The mean cumulative incidence of NVA-HARTI was 7.2 (6.4-8.0) per 100 ICU admissions that did not change significantly over time ( $p$-value $=0.72$ ). The mean cumulative incidence of VA-HARTI was 15.4 (14.2-16.5) per 100 ICU admissions; it significantly reduced from 19.0 (14.6-23.4) in 2011 to 11.7 (8.714.6) in 2020, p-value $=0.008$ (Fig. $2 A$ ).

The mean incidence rate of NVA-HARTI was $4.2 \pm 2.0$ per 1000 patient-days in the ICU with no time trend, $p$-value $=0.15$. The mean incidence rate of VA-HARTI was $9.5 \pm 3.0$ per 1000 patient-days, also with no time trend, p-value $=0.09$, Fig. 2B.

When normalized to ventilator-days, VA-HARTI incidence rate didn't change throughout the study period ( $p$ value $=0.79)$ and averaged at $16.4 \pm 4.7$ cases per 1000 ventilator-days (Fig. $2 \mathrm{C}$ ). At the same time, the total number of ventilator-days per year decreased by 30\%, from 3,690 in 2011 to 2,565 in 2020.

\section{Length of Stay}

NVA-HARTI group had a significantly higher median hospital LOS (32 days [21; 48.5]) comparing to No HAI (17 days [11; 25]), though it was significantly shorter than in the VA-HARTI group, 46 days [28; 76.5], Fig. 3A. From 2011 to 2020, hospital LOS did not change in the NVA-HARTI group although it decreased in all other groups. Additionally, the variance of hospital LOS decreased in all groups (Figure S3A).

The ICU LOS in the NVA-HARTI group was 15 days [10; 28.75], significantly higher than in No HAI and Other HAI groups. The longest ICU LOS was in the VA-HARTI group with a median of 26 days [17; 43]; the shortest ICU LOS was in No HAI group with a median of 5 days [3; 7], Fig. 3B. Over time, the ICU LOS remained unchanged for both HARTI groups but decreased significantly for all other groups (Figure S3B).

\section{Mortality Rates}


The mean all-cause in-hospital mortality in the study population was $10.8 \%(9.8-11.8)$ over ten years. It declined significantly from $16.4 \%(12.2-21)$ in 2011 to $5.4 \%(3.1-7.9)$ in 2020 , p-value $=0.0001$ (Fig. 3E).

In the NVA-HARTI group, the mortality rate was $12.3 \%$ (7.9-16.8), and no different from other groups. In VA-HARTI patients, the mortality rate was $16.7 \%$ (13.6-19.7), twice that of the No HAI group, $8.2 \%$ (7.09.4), p-value $<0.0001$, Fig. 4C. From 2011 to 2020 , the mortality rate dropped significantly in all groups except for the NVA-HARTI, Figure S3C.

\section{Survival Analysis and the Impact of HARTI on Mortality}

Patients with NVA-HARTI, VA-HARTI, and with no HAl died on median hospital days 35 [12.5; 57], 35.5 [18; 75.25], and 10 [6; 18] respectively, Figure S4A. This difference represented the immortal time bias. To account for it, we adjusted for time-independent variables (age, sex, diagnosis, and CCI) in a Cox time varying model. Neither infection was found to be independently associated with mortality, NVA-HARTI pvalue $=0.13$ (Table S5A), VA-HARTI p-value $=0.82$ (Table S5B).

Survival curves were obtained from the competing risk model while accounting for discharge as a competing event, Fig. 3D. The Kaplan-Meier model underestimated the risk of death and was deemed inappropriate (Figure S4B). The survival probability difference function showed that during the first 20 days in the hospital, patients in the No HAl group had a significantly higher chance of dying than patients in both HARTI groups. Later on, survival curves crossed swapping the risk between groups: No HAl and VA-HARTI at approximately hospital day 30 and No HAI and NVA-HARTI at approximately hospital day 45 . After hospital day 52, patients in both HARTI groups had higher probability of death and this equilibrium continued up to hospital day 228 when the last patient in the No HAl group was discharged or died (Fig. 3D).

In stepwise logistic regression, VA-HARTI was an independent risk factor of death with an odds ratio of 1.54 (1.11-2.14), p-value $=0.009$ (Table 2A, all covariates in Table S6A). NVA-HARTI was not a predictor of mortality, odds ratio $1.43(0.99-2.06)$, $p$-value $=0.057$ (Table 2B, all covariates in Table S6B). The performance testing in five-fold cross-validation yielded a ROC-AUC score of 0.63 for NVA-HARTI and 0.79 for VA-HARTI models. 
Table 2A

Multivariate logistic regression evaluating mortality in all study patients; the estimate for VA-HARTI. Only significant covariates are shown, for full data table refer to Table S6A. Abbreviations: CSFL-NE, CSF leakage from nose and/or ears; ICU, intensive care unit; SSSI, superficial surgical site infections; VA-HARTI, ventilator-associated healthcare-associated respiratory tract infections.

\begin{tabular}{|c|c|c|c|c|}
\hline Factor & $\begin{array}{l}\text { Odds } \\
\text { ratio }\end{array}$ & $\begin{array}{l}\text { Lower band of } \\
95 \% \mathrm{Cl}\end{array}$ & $\begin{array}{l}\text { Upper band of } \\
95 \% \mathrm{Cl}\end{array}$ & $\begin{array}{l}\text { p- } \\
\text { value }\end{array}$ \\
\hline Days with bloodstream infection & 1.07 & 1.01 & 1.12 & 0.0136 \\
\hline Days with intestinal dysfunction & 1.03 & 1.01 & 1.05 & 0.0041 \\
\hline Days with SSSI & 1.02 & 1.00 & 1.03 & 0.0087 \\
\hline Days with tracheostomy & 0.96 & 0.94 & 0.98 & 0.0001 \\
\hline Days with urinary catheter & 1.02 & 1.00 & 1.05 & 0.0430 \\
\hline Days with vasopressors & 1.02 & 1.00 & 1.04 & 0.0379 \\
\hline Glasgo Coma Scale & 0.59 & 0.57 & 0.62 & $\begin{array}{l}< \\
0.0001\end{array}$ \\
\hline Patients undergoing craniotomy & 0.73 & 0.59 & 0.88 & 0.0015 \\
\hline Patients with brain tumors & 1.86 & 1.38 & 2.51 & $\begin{array}{l}< \\
0.0001\end{array}$ \\
\hline Patients with CSFL-NE & 1.95 & 1.08 & 3.50 & 0.0259 \\
\hline Patients with persistent vegetative state & 0.27 & 0.16 & 0.44 & $\begin{array}{l}< \\
0.0001\end{array}$ \\
\hline Patients with traumatic brain injuries & 0.44 & 0.26 & 0.75 & 0.0024 \\
\hline $\begin{array}{l}\text { The total number of all surgeries during } \\
\text { one ICU admission }\end{array}$ & 1.20 & 1.06 & 1.36 & 0.0039 \\
\hline VA-HARTI & 1.54 & 1.11 & 2.14 & 0.0090 \\
\hline
\end{tabular}


Multivariate logistic regression evaluating mortality in all study patients; the estimate for NVA-HARTI. Only significant covariates are shown, for full data table refer to Table S6B. Abbreviations: CSFL-NE, CSF leakage from nose and/or ears.

\begin{tabular}{|lllll|}
\hline Factor & $\begin{array}{l}\text { Odds } \\
\text { ratio }\end{array}$ & $\begin{array}{l}\text { Lower band of 95\% } \\
\text { Cl }\end{array}$ & $\begin{array}{l}\text { Upper band of 95\% } \\
\text { Cl }\end{array}$ & p-value \\
\hline Days with antibiotics & 0.97 & 0.94 & 0.99 & 0.0072 \\
\hline Days with central line & 1.02 & 1.00 & 1.04 & 0.0323 \\
\hline $\begin{array}{l}\text { Days with intestinal } \\
\text { dysfunction }\end{array}$ & 1.03 & 1.01 & 1.05 & 0.0011 \\
\hline Days with urinary catheter & 1.03 & 1.01 & 1.06 & 0.0014 \\
\hline Days with vasopressors & 1.04 & 1.02 & 1.06 & $<0.0001$ \\
\hline Patients with brain tumors & 1.75 & 1.36 & 2.25 & $<0.0001$ \\
\hline Patients with CSFL-NE & 1.86 & 1.32 & 2.62 & 0.0004 \\
\hline
\end{tabular}

\section{Risk of HARTI}

The risk of both HARTI types increased rapidly in the beginning of ICU stay. The NVA-HARTI probability grew faster and plateaued earlier than VA-HARTI, around day 8 in the ICU with the highest daily risk of $1.0 \%$ at that time. Whereas the risk of VA-HARTI continued to increase up to ICU day 20 before reaching a plateau phase (Fig. 4A). Also, a secondary peak of daily infection risk occurred earlier for NVA-HARTI, Fig. 4B. During the first week in the ICU, the risk of infection doubled every 18.4 hours for NVA-HARTI and every 13.6 hours for VA-HARTI.

The cumulative probability of VA-HARTI depending on the number of days on mechanical ventilation increased rapidly during the first 8 ventilation days, Fig. 4C. The probability of VA-HARTI doubled daily during the first week on ventilation. A secondary peak became apparent after ventilation day 36 (Fig. 4D).

\section{Discussion}

A prospective observational study was performed at a large tertiary referral neurosurgical center to examine the incidence and outcomes associated with NVA-HARTI and to compare it to VA-HARTI. The main findings of this study are: 1) both VA-and NVA-HARTI are prevalent in the neurosurgical ICU however they are different in terms of incidence, prevalence, and risks, 2) while the VA-HARTI incidence reduced over time with preventive measures in place, NVA-HARTI incidence did not change, 3) NVA-HARTI was associated with higher hospital and ICU LOS, but not with higher mortality compared to patients without HAI, and 4) VA-HARTI was an independent predictor of mortality while NVA-HARTI was not. We 
demonstrated that NVA-HARTI is a distinct clinical entity that persists despite preventive measures and is associated with poor outcomes.

\section{The Rate of Occurrence of NVA and VA-HARTI}

To our knowledge, the incidence of HARTI in non-ventilated neurosurgical ICU patients is unknown and comprehensive epidemiological data on NVA-HARTI in ICU is limited (4). The most comparable study was done in Europe on mixed ICU patients who stayed $>48 \mathrm{~h}$ in the ICU; it reported $1 \%$ incidence of nonventilator pneumonia (7). In our study, the cumulative incidence of NVA-HARTI was significantly higher, $7.2 \%$. The difference can be explained by higher prevalence of factors predisposing neurosurgical patients to respiratory infections. The incidence rate in the mixed hospital population was 3.63 per 1000 patient-days in the USA (15) which is similar to our result, 4.2 per 1000 patient-days.

Notably, the cumulative incidence of NVA-HARTI did not change over the ten-year study period. As previously reported, the incidence of pneumonia remained stable for 5 years in acute-care non-ventilated patients (16).

In comparison, the cumulative incidence of VA-HARTI in our study was comparable to previous reports on VAP in neurosurgical ICU patients, $15.4 \%$ (17). However, in literature, the incidence varies significantly $(2.3-50.7 \%)$ depending on the specific neurosurgical procedure and patient characteristics $(18,19)$. For example, in patients with traumatic brain injuries, VAP incidence was found to be $36 \%(20)$.

The incidence rate of VA-HARTI in our study was 16.4 per 1000 ventilator-days which is within the range of previous European reports of 19.0 per 1000 ventilator-days (21) and 11.0 per 1000 ventilator-days (22).

\section{Outcomes in Patients with NVA and VA-HARTI}

There is an emphasis in literature that the resulting burden of NVA-HARTI on patient outcomes in neurocritical care is not fully known (20), and our primary aim was to report hospital and ICU LOS and mortality in this patient population. Our results are consistent with previously reported outcomes from mixed ICU in Spain (23). In our study, the ICU LOS was significantly higher in NVA-HARTI patients than in patients with no HAI. Such a difference was also reported before (23). Of note, the differences in LOS between patients with and without HARTI should be interpreted with caution as a number of baseline characteristics were different between groups (Table S3).

Whereas no available publications examine the mortality in neurosurgical ICU patients with NVA-HARTI, in mixed ICU population, mortality rate in non-ventilated patients with pneumonia was reported to be $23 \%$ within the 30 days of pneumonia onset (7). A 2010 mixed ICU study reported $36 \%$ hospital mortality in non-ventilator ICU-acquired pneumonia patients (23). Both rates are higher than those observed in our study. These studies included only patients with pneumonia, the most severe type of HARTI, while we included all respiratory infections that may ultimately skew the results toward lower mortality. Additionally, the overall mortality in our study population was lower $(10.8 \%)$ than previously reported in similar neurosurgical ICUs, $19.0 \%$ (22). Thus, lower NVA-HARTI mortality could be a reflection of lower 
overall mortality. Death rate in VA-HARTI patients was $26 \%$ higher than in NVA-HARTI patients. However, it was lower than reported previously for neurosurgical ICU patients with VAP, $28.4 \%$ (7). Of note, the mortality in patients with VAP in general ICU populations varies considerably across studies (4), and the latest European data suggests a rate of $37.7 \%(24)$.

In this study, NVA-HARTI was not associated with increased mortality while VA-HARTI independently increased chances of death by $54 \%$. There is an ongoing discussion about the impact of VAP on mortality with controversial evidence (25). A 2017 study on ICU patients with intracerebral hemorrhage found that VAP independently increased in-hospital mortality, odds ratio 2.68 (2.58-2.77) (17). Similarly, a 2019 study in French mixed ICUs found that VAP significantly increased mortality by $38 \%$ (7). Conversely, a meta-analysis in patients with traumatic brain injury did not find an association between VAP and mortality (20). Another study on patients in neurologic ICU also did not show that VAP influenced mortality, but in that study the absolute number of patients who died in the VAP group and in relation to VAP was very low (26). When obtained from randomized prevention studies, a meta-analysis estimated an attributable VAP mortality to be $13 \%$ (27). Accounting for preexisting differences and evolution of the risk of death over time yielded an attributable mortality of $2.3 \%$ per day with VAP (28). The controversy around VAP impact on mortality can be due to a complex time-dependent balance of multiple factors contributing to overall risk.

\section{Risks of NVA-HARTI and Comparison with VA-HARTI}

We found that the risk of NVA-HARTI increased with increasing ICU stay in a non-linear manner. The highest daily risk was in the beginning of the ICU course followed by a smaller peak later on. A similar result was demonstrated previously for VAP (29). The fact that the risk is uneven throughout the ICU stay can be a basis for developing a flexible prevention strategy with time-dependent preventive measures.

The risk of HARTI over time was different in ventilated and non-ventilated patients. NVA-HARTI occurred earlier in the course of ICU stay and exhibited a shorter disease course compared to VA-HARTI. This result is consistent with previously published data (16). Understanding risks of HARTI and specifically the dynamics of risk in time can help guide development of prevention measures.

\section{Strengths, Limitations and Further Directions}

The strength of our study is the carefully curated and prospectively collected database that included a large cohort of neurosurgical ICU patients and many years of observation. Long period of observation allowed for a reliable estimate of infection and mortality rates. We utilized the method of survival analysis that was the most suitable for the specifics of HAI in neurosurgical ICU and accounted for immortal time bias. This study also has some limitations including its observational nature and single-center settings. Additionally, the definition of HARTI was based on clinical parameters and subjective complicating comparisons to other studies. Accordingly, HARTI events may have also been tracheobronchitis or laryngitis episodes that may have otherwise overestimated infection rates and underestimated mortality rates. The metric of crude in-hospital mortality and lack of follow-up after the discharge also limited the 
ability to completely assess the impact of HARTI on mortality. Future multicenter studies may provide better estimates of the effect of HARTI on patient outcomes.

\section{Conclusion}

Our findings suggest that NVA-HARTI in neurosurgical ICU patients represents a significant healthcare burden with relatively high incidence and associated poor outcomes. NVA-HARTI appears to be different from VA-HARTI and persisted despite preventive measures; therefore, extrapolating VA-HARTI research findings to NVA-HARTI should be avoided.

\section{List Of Abbreviations}

\begin{tabular}{|ll|}
\hline ADF & Augmented Dickey-Fuller test; \\
\hline CDC/NHSN & The Center for Disease Control / National Healthcare Safety Network; \\
\hline CI & Confidence interval; \\
\hline CNS & Central Nervous System; \\
\hline ICU & Intensive Care Unit; \\
\hline ICU LOS & Length of Stay in the ICU; \\
\hline HAI & Healthcare-Associated Infections; \\
\hline HARTI & Healthcare-Associated Respiratory Tract Infections; \\
\hline LOS & Length of Stay in the hospital; \\
\hline NVA-HARTI & Non-Ventilator-Associated Healthcare-Associated Respiratory Tract Infections; \\
\hline NSI & Burdenko National Medical Research Center of Neurosurgery; \\
\hline POA & Present on Admission; \\
\hline ROC-AUC & Receiving-Operating Curve, an Area Under the Curve; \\
\hline VA-HARTI & Ventilator-Associated Healthcare-Associated Respiratory Tract Infections; \\
\hline VAP & Ventilator-Associated Pneumonia; \\
\hline
\end{tabular}

\section{Declarations}

\section{Ethics approval and consent to participate}

The NSI Institutional Review Board approved this study. An informed consent waiver was obtained from the Institutional Review Board based on the study's observational nature. All data was anonymized before data analysis. 


\section{Consent for publication}

Not applicable.

\section{Availability of data and materials}

The full code for statistical analysis is available at https://github.com/KseniaErshova/HARTI, The dataset supporting the conclusions of this article is available in the Zenodo repository, https://doi.org/10.5281/zenodo.3995319.

\section{Competing interests}

The authors declare that they have no competing interests.

\section{Funding}

None reported.

\section{Authors' contribution}

$\mathrm{KE}, \mathrm{OK}, \mathrm{DW}$, and $\mathrm{AL}$ analyzed data and wrote the manuscript; OE and IS developed the study design, implemented and maintained the infection prevention program in the ICU; GD, IS and ES collected data, evaluated and treated study subjects; MS developed and supported electronic surveillance protocol. All authors read and approved the final manuscript.

\section{Acknowledgement}

We'd like to acknowledge the contribution of Burdenko Neurosurgery Institute clinicians, nurses, and administrators who helped implement the infection control program in the ICU and participated in data collection. Special thanks to Dr. Philip Lumb, Dr. Holly Muir, Mary Bading (University of Southern California) for their insight, expertise and guidance.

\section{References}

1. Kalanuria AA, Ziai W, Mirski M. Ventilator-associated pneumonia in the ICU. Crit Care [Internet]. 2014 Mar 18;18(2):208. Available from: http://dx.doi.org/10.1186/cc13775

2. Heyland DK, Cook DJ, Griffith L, Keenan SP, Brun-Buisson C. The attributable morbidity and mortality of ventilator-associated pneumonia in the critically ill patient. The Canadian Critical Trials Group. Am J Respir Crit Care Med [Internet]. 1999 Apr;159(4 Pt 1):1249-56. Available from:

http://dx.doi.org/10.1164/ajrccm.159.4.9807050 
3. Klompas M. Hospital-Acquired Pneumonia in Nonventilated Patients: The Next Frontier. Infect Control Hosp Epidemiol [Internet]. 2016 Jul;37(7):825-6. Available from: http://dx.doi.org/10.1017/ice.2016.101

4. Ferrer M, Torres A. Epidemiology of ICU-acquired pneumonia. Curr Opin Crit Care [Internet]. 2018 Oct;24(5):325-31. Available from: http://dx.doi.org/10.1097/MCC.0000000000000536

5. Ewan VC, Witham MD, Kiernan M, Simpson AJ. Hospital-acquired pneumonia surveillance-an unmet need. Lancet Respir Med [Internet]. 2017 Oct;5(10):771-2. Available from: http://dx.doi.org/10.1016/S2213-2600(17)30296-5

6. Magill SS, Edwards JR, Bamberg W, Beldavs ZG, Dumyati G, Kainer MA, et al. Multistate pointprevalence survey of health care-associated infections. N Engl J Med [Internet]. 2014 Mar 27;370(13):1198-208. Available from: http://dx.doi.org/10.1056/NEJMoa1306801

7. Ibn Saied W, Mourvillier B, Cohen Y, Ruckly S, Reignier J, Marcotte G, et al. A Comparison of the Mortality Risk Associated With Ventilator-Acquired Bacterial Pneumonia and Nonventilator ICUAcquired Bacterial Pneumonia. Crit Care Med [Internet]. 2019 Mar;47(3):345-52. Available from: http://dx.doi.org/10.1097/CCM.0000000000003553

8. Saied WI, Martin-Loeches I, Timsit J-F. What is new in non-ventilated ICU-acquired pneumonia? Intensive Care Med [Internet]. 2020 Mar;46(3):488-91. Available from: http://dx.doi.org/10.1007/s00134-019-05859-9

9. Center for Disease Control. Identifying healthcare-associated infections [Internet]. 2018. Available from: https://www.cdc.gov/nhsn/PDFs/pscManual/2PSC_IdentifyingHAls_NHSNcurrent.pdf

10. Horan TC, Andrus M, Dudeck MA. CDC/NHSN surveillance definition of health care-associated infection and criteria for specific types of infections in the acute care setting. Am J Infect Control [Internet]. 2008 Jun;36(5):309-32. Available from: http://dx.doi.org/10.1016/j.ajic.2008.03.002

11. Fine JP, Gray RJ. A Proportional Hazards Model for the Subdistribution of a Competing Risk. J Am Stat Assoc [Internet]. 1999 Jun 1;94(446):496-509. Available from: https://www.tandfonline.com/doi/abs/10.1080/01621459.1999.10474144

12. Gray B. cmprsk: Subdistribution Analysis of Competing Risks [Internet]. 2019. Available from: https://CRAN.R-project.org/package= $=$ cmprsk

13. Seabold S, Perktold J. Statsmodels: Econometric and statistical modeling with python. In: Proceedings of the 9th Python in Science Conference [Internet]. SciPy; 2010. Available from: https://conference.scipy.org/proceedings/scipy2010/seabold.html

14. Jones E, Oliphant T, Peterson P. SciPy: Open source scientific tools for Python. 2001-; Available from: http://www.scipy.org

15. Giuliano KK, Baker D, Quinn B. The epidemiology of nonventilator hospital-acquired pneumonia in the United States. Am J Infect Control [Internet]. 2018 Mar;46(3):322-7. Available from: http://dx.doi.org/10.1016/j.ajic.2017.09.005

16. Strassle PD, Sickbert-Bennett EE, Klompas M, Lund JL, Stewart PW, Marx AH, et al. Incidence and risk factors of non-device-associated pneumonia in an acute-care hospital. Infect Control Hosp Epidemiol 
[Internet]. 2020 Jan;41(1):73-9. Available from: http://dx.doi.org/10.1017/ice.2019.300

17. Murthy SB, Moradiya Y, Shah J, Merkler AE, Mangat HS, ladacola C, et al. Nosocomial Infections and Outcomes after Intracerebral Hemorrhage: A Population-Based Study. Neurocrit Care [Internet]. 2016 Oct;25(2):178-84. Available from: http://dx.doi.org/10.1007/s12028-016-0282-6

18. Jovanovic B, Milan Z, Markovic-Denic L, Djuric O, Radinovic K, Doklestic K, et al. Risk factors for ventilator-associated pneumonia in patients with severe traumatic brain injury in a Serbian trauma centre. Int J Infect Dis [Internet]. 2015 Sep;38:46-51. Available from: http://dx.doi.org/10.1016/j.ijid.2015.07.005

19. Abulhasan YB, Rachel SP, Châtillon-Angle M-O, Alabdulraheem N, Schiller I, Dendukuri N, et al. Healthcare-associated infections in the neurological intensive care unit: Results of a 6-year surveillance study at a major tertiary care center. Am J Infect Control [Internet]. 2018 Jun [cited 2019 Mar 6];46(6):656-62. Available from: http://dx.doi.org/10.1016/j.ajic.2017.12.001

20. Li Y, Liu C, Xiao W, Song T, Wang S. Incidence, Risk Factors, and Outcomes of Ventilator-Associated Pneumonia in Traumatic Brain Injury: A Meta-analysis. Neurocrit Care [Internet]. 2020 Feb;32(1):27285. Available from: http://dx.doi.org/10.1007/s12028-019-00773-w

21. Martin-Loeches I, Povoa P, Rodríguez A, Curcio D, Suarez D, Mira J-P, et al. Incidence and prognosis of ventilator-associated tracheobronchitis (TAVeM): a multicentre, prospective, observational study. Lancet Respir Med [Internet]. 2015 Nov;3(11):859-68. Available from: http://dx.doi.org/10.1016/S2213-2600(15)00326-4

22. Orsi GB, Scorzolini L, Franchi C, Mondillo V, Rosa G, Venditti M. Hospital-acquired infection surveillance in a neurosurgical intensive care unit. J Hosp Infect [Internet]. 2006 Sep;64(1):23-9. Available from: http://dx.doi.org/10.1016/j.jhin.2006.02.022

23. Esperatti M, Ferrer M, Theessen A, Liapikou A, Valencia M, Saucedo LM, et al. Nosocomial pneumonia in the intensive care unit acquired by mechanically ventilated versus nonventilated patients. Am J Respir Crit Care Med [Internet]. 2010 Dec 15;182(12):1533-9. Available from: http://dx.doi.org/10.1164/rccm.201001-00940C

24. Koulenti D, Tsigou E, Rello J. Nosocomial pneumonia in 27 ICUs in Europe: perspectives from the EUVAP/CAP study. Eur J Clin Microbiol Infect Dis [Internet]. 2017 Nov;36(11):1999-2006. Available from: http://dx.doi.org/10.1007/s10096-016-2703-z

25. Bregeon F, Ciais V, Carret V, Gregoire R, Saux P, Gainnier M, et al. Is ventilator-associated pneumonia an independent risk factor for death? Anesthesiology [Internet]. 2001 Apr;94(4):554-60. Available from: http://dx.doi.org/10.1097/00000542-200104000-00005

26. Josephson SA, Moheet AM, Gropper MA, Nichols AD, Smith WS. Ventilator-associated pneumonia in a neurologic intensive care unit does not lead to increased mortality. Neurocrit Care [Internet]. 2010 Apr;12(2):155-8. Available from: http://dx.doi.org/10.1007/s12028-009-9285-x

27. Melsen WG, Rovers MM, Groenwold RHH, Bergmans DCJJ, Camus C, Bauer TT, et al. Attributable mortality of ventilator-associated pneumonia: a meta-analysis of individual patient data from 
randomised prevention studies. Lancet Infect Dis [Internet]. 2013 Aug;13(8):665-71. Available from: http://dx.doi.org/10.1016/S1473-3099(13)70081-1

28. Bekaert M, Timsit J-F, Vansteelandt S, Depuydt P, Vésin A, Garrouste-Orgeas M, et al. Attributable mortality of ventilator-associated pneumonia: a reappraisal using causal analysis. Am J Respir Crit Care Med [Internet]. 2011 Nov 15;184(10):1133-9. Available from:

http://dx.doi.org/10.1164/rccm.201105-08670C

29. Jaimes F, De La Rosa G, Gómez E, Múnera P, Ramírez J, Castrillón S. Incidence and risk factors for ventilator-associated pneumonia in a developing country: where is the difference? Respir Med [Internet]. 2007 Apr;101(4):762-7. Available from: http://dx.doi.org/10.1016/j.rmed.2006.08.008

\section{Figures}




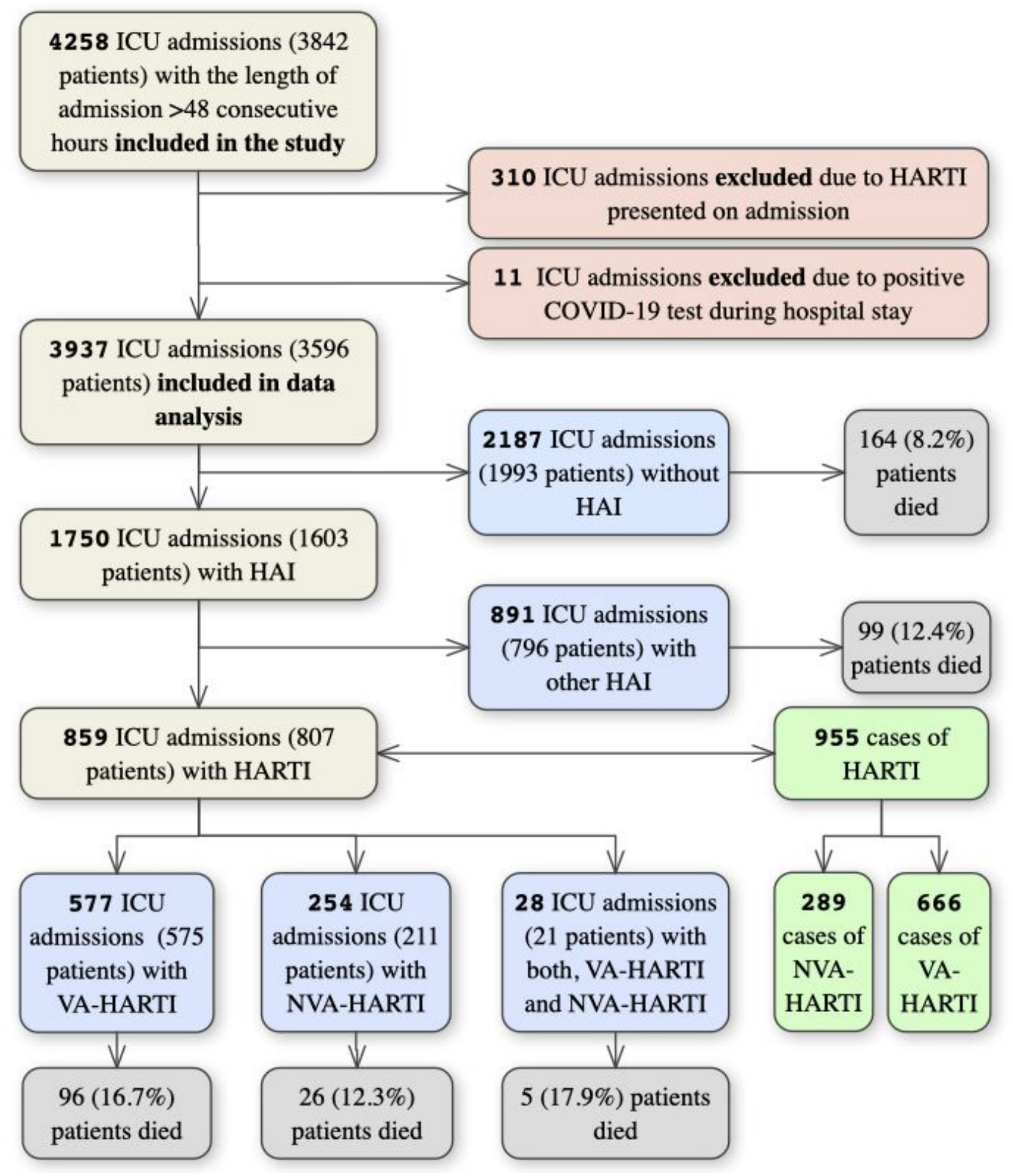

Figure 1

A flow chart showing the process of patient selection in the study with the number of patients, ICU admissions, cases and number of deaths per group. Abbreviations: ICU, intensive care unit; HARTI, healthcare-associated respiratory tract infection; NVA-HARTI, non-ventilator associated healthcareassociated respiratory tract infections; VA-HARTI, ventilator-associated healthcare-associated respiratory tract infections. 

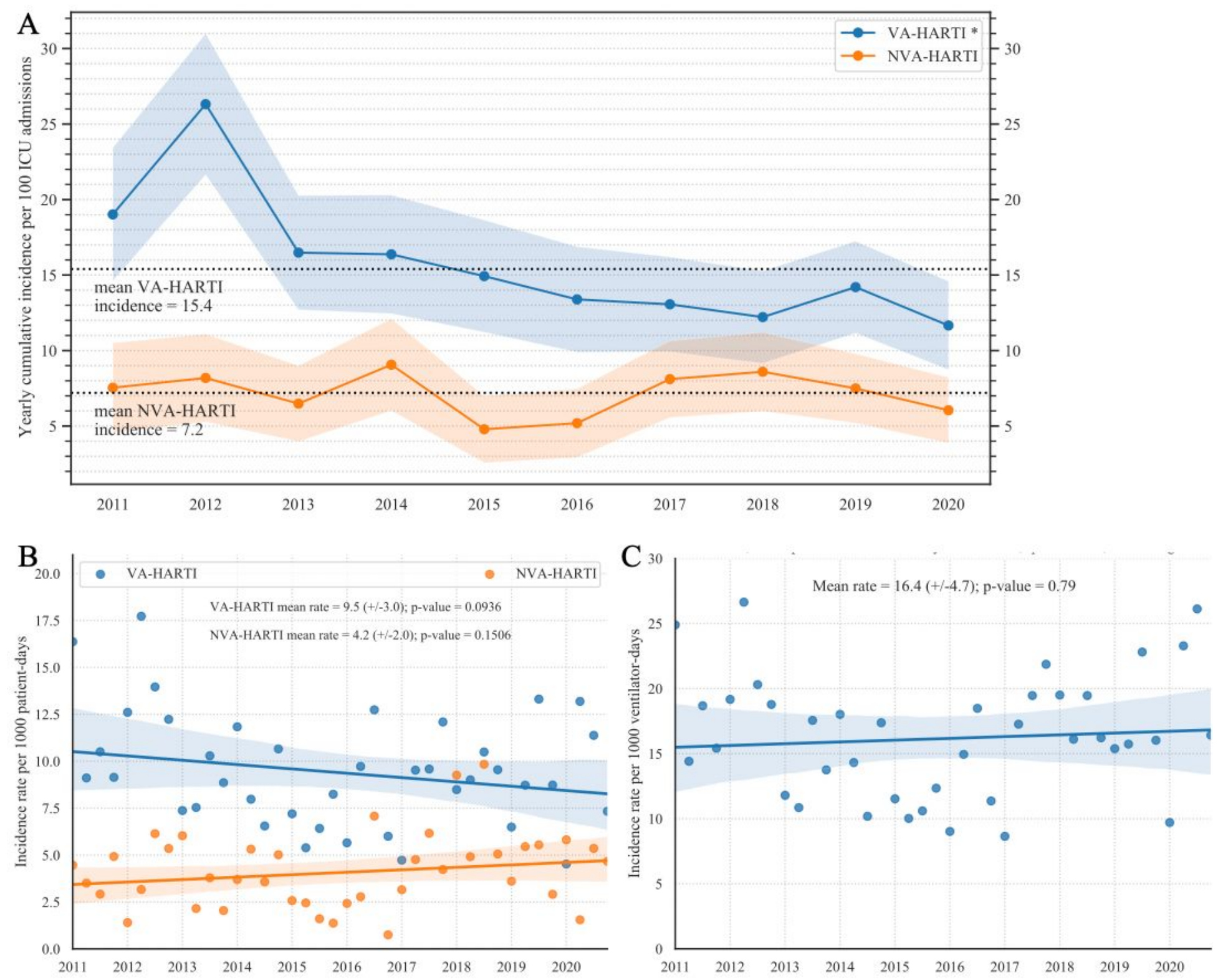

\section{Figure 2}

Incidence of HARTI in neurosurgical ICU patients. A, yearly cumulative incidence of HARTI per 100 ICU admissions with $95 \%$ confidence interval for binomial distribution. The mean incidence during the study period shown as a dotted line; B, incidence rates of VA- and NVA-HARTI per 1000 patient-days in the ICU; C, incidence rate of VA-HARTI per 1000 ventilator-days. Star $\left(^{*}\right)$ in the legend indicates groups with statistically significant time trend. In $B$ and $C$ we used incidence rate per quarter; regression line and $p$ values obtained from the robust linear regression. Abbreviations: ICU, intensive care unit; NVA-HARTI, nonventilator-associated healthcare-associated respiratory tract infections; VA-HARTI, ventilator-associated healthcare-associated respiratory tract infections. 

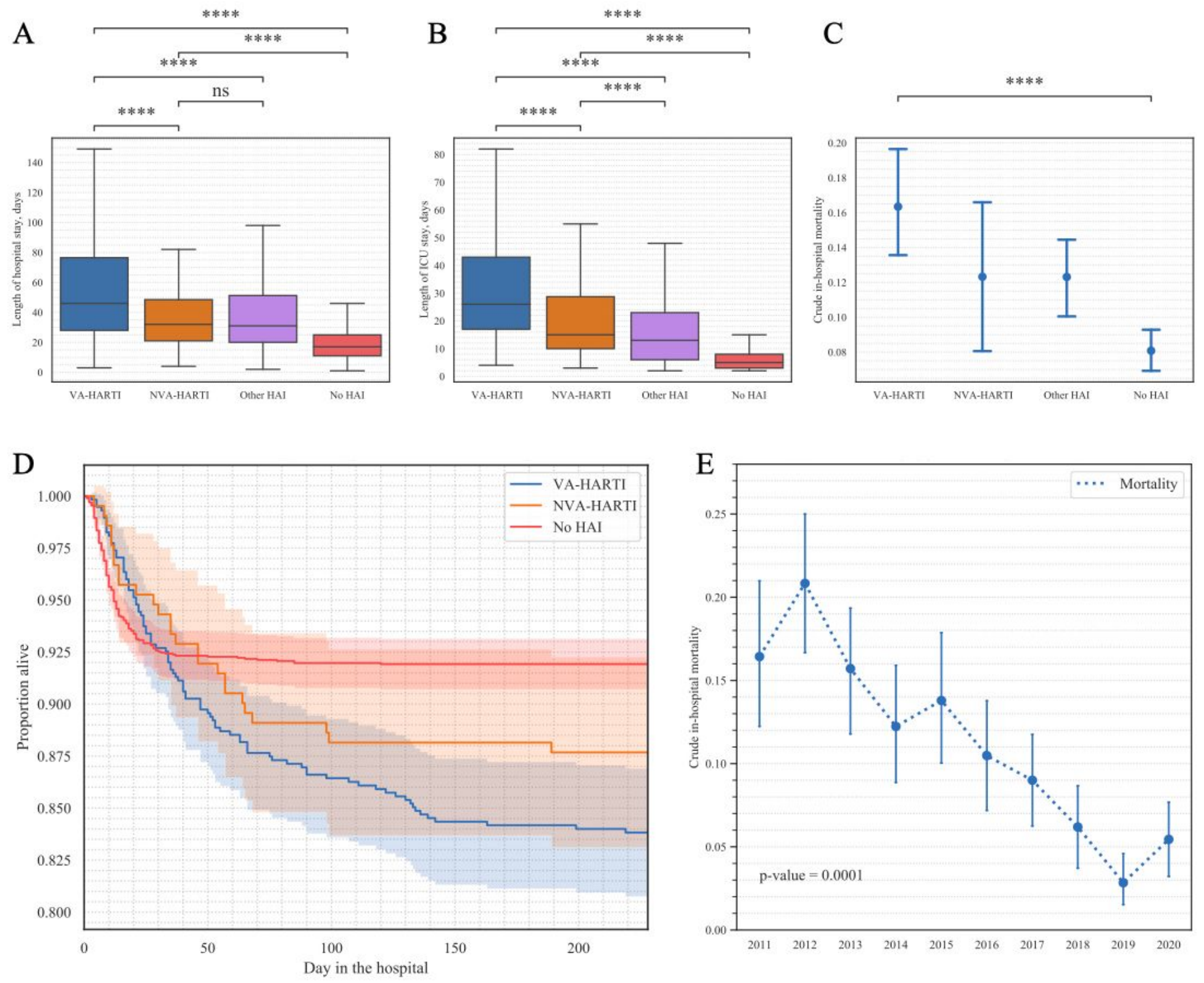

Figure 3

Clinical outcomes in different groups of neurosurgical ICU patients including VA-HARTI, NVA-HARTI, Other HAI (including CNS infections, urinary tract infections, bloodstream infections, and superficial surgical site infections), and No HAI groups. A, median hospital length of stay; B, median ICU length of stay; C, crude in-hospital mortality per 100 patients in the group; D, survival probability while accounting for discharge as a competing event (competing risk model); $E$, crude in-hospital mortality in the entire study population from 2011 to 2018. In figures A and B, p-values obtained from Kruskal-Wallis test with post-hoc pairwise testing performed by Conover test; $p$-value annotation legend: $n s:>0.05 ; *: 0.01<p \leqq 0.05 ; * \star: 0.001<p \leqq$ $0.01 ; * \star *: 0.0001<p \leqq 0.001$; $* \star \star *: p<0.0001$. Abbreviations: ICU, intensive care unit; HAl, healthcareassociated infections; NVA-HARTI, non-ventilator-associated healthcare-associated respiratory tract infections; VA-HARTI, ventilator-associated healthcare-associated respiratory tract infections. 

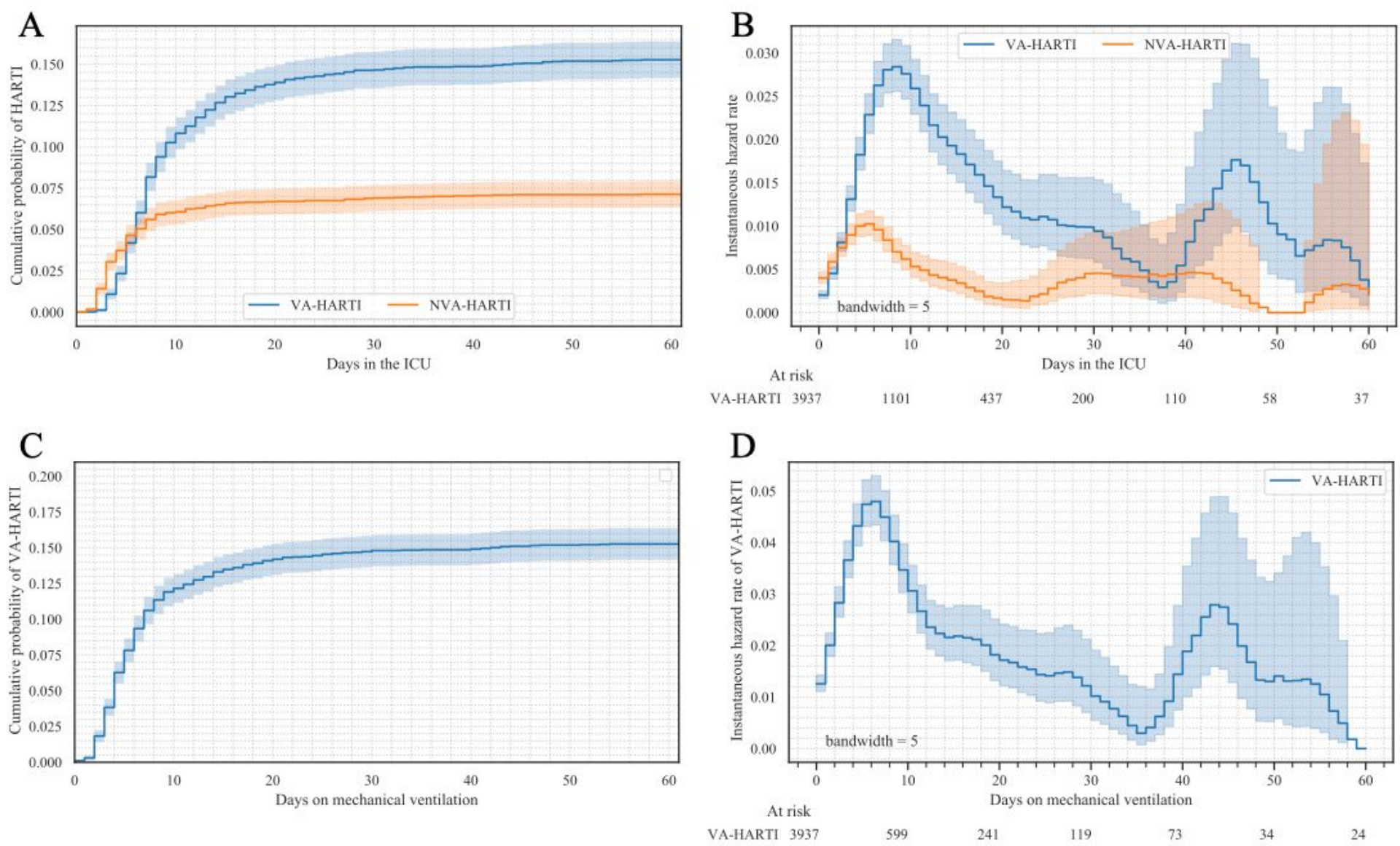

Figure 4

Risk of HARTI. Set 1 (A and C), the cumulative probability of HARTI based on competing event regression model that accounted for a discharge from the ICU and death as competing events. A, probability of HARTI depending on the number of days in the ICU; $C$, probability of VA-HARTI depending on the number of days on mechanical ventilation. Set 2 ( $B$ and $D$ ), the kernel-smoothed function of instantaneous hazard based on a Nelsen-Aalen estimate. B, instantaneous hazard of HARTI depending on the number of days in the ICU; D, instantaneous hazard of VA-HARTI depending on the number of days on mechanical ventilation. Abbreviations: NVA-HARTI, non-ventilator-associated healthcare-associated respiratory tract infections; VA-HARTI, ventilator-associated healthcare-associated respiratory tract infections.

\section{Supplementary Files}

This is a list of supplementary files associated with this preprint. Click to download.

- HARTISupplementwide.pdf 